\title{
BMJ Open Shock wave therapy associated with eccentric strengthening versus isolated eccentric strengthening for Achilles insertional tendinopathy treatment: a double-blinded randomised clinical trial protocol
}

\author{
Nacime Salomão Barbachan Mansur, ${ }^{1}$ Flávio Faloppa, ${ }^{2}$ João Carlos Belloti, ${ }^{2}$ \\ Sheila J McNeill Ingham, ${ }^{1}$ Fabio Teruo Matsunaga, ${ }^{2}$ Paulo Roberto Dias dos Santos, ${ }^{1}$ \\ Bruno Schiefer dos Santos, ${ }^{1}$ Oreste Lemos Carrazzone, ${ }^{1}$ Gabriel Peixoto, ${ }^{1}$ \\ Bruno Takeshi Aoyama, ${ }^{1}$ Marcel Jun Sugawara Tamaoki ${ }^{1}$
}

To cite: Mansur NSB, Faloppa F, Belloti JC, et al. Shock wave therapy associated with eccentric strengthening versus isolated eccentric strengthening for Achilles insertional tendinopathy treatment: a double-blinded randomised clinical trial protocol. BMJ Open 2017;7:e013332. doi:10.1136/bmjopen-2016013332

- Prepublication history for this paper is available online. To view these files please visit the journal online (http://dx.doi.org/10.1136/ bmjopen-2016-013332).

Received 13 July 2016 Revised 14 December 2016 Accepted 20 December 2016

CrossMark

For numbered affiliations see end of article.

Correspondence to Dr Nacime Salomão Barbachan Mansur; nacime@uol.com.br

\section{ABSTRACT}

Background: There is no consensus regarding the treatment of Achilles insertional tendinopathies. Eccentric training remains the main choice in the conservative treatment of this illness; however, the good results in the management of non-insertional Achilles tendinopathy were not replicated in the insertional condition. Low energy shock wave therapy has been described as an alternative to these patients, but has yet to be empirically tested.

Hypothesis: Shock wave therapy, adjunctive to the eccentric strengthening protocol, will improve measures of pain and function.

Design: Double blind, placebo-controlled, parallel groups, randomised clinical trial.

\section{Materials and methods: 93 patients with a} diagnosis of chronic insertional tendinopathy, referred from primary or secondary healthcare services, will be assessed and enrolled in this study. They will be divided into two groups (randomised by sequentially numbered identical envelopes, which will be administered serially to participants), one containing the combination of low energy shock wave and eccentric exercises, as treatment and the other comprehending the exercises and the placebo treatment (an apparatus placed in the therapeutic head). The assessments will occur in 2, 4, 6, 12 and 24 weeks. Patients will be evaluated primarily by the Victorian Institute of Sport Assessment-Achilles questionnaire and secondarily by the visual analogue scale, Algometry, the American Orthopedic Foot and Ankle Society scale, the Foot and Ankle Outcome Score and the 12-item Short Form Health Survey. We will use comparison of two proportions via relative frequency analysis, the Pearson Correlation the $\chi^{2}$ test and the analysis of variance for statistical analyses.

Discussion: This study intends to demonstrate if the association of the eccentric exercise programme with the shock wave therapy can produce good results regarding

\section{Strengths and limitations of this study}

- Study design is ideal for treatment recommendations.

- Combination of methods novelty.

- Previous sample size calculation.

- Double-blinded design minimising benchmarking bias.

- Previous protocol publication minimising publication bias.

- Short follow-up course limiting the cross-check of long-term effects and complications.

- Single-centre study decreasing external validation.

the treatment of the Achilles insertional tendinopathy. In an attempt to prevent the high costs and complications associated with the surgical intervention, we will try to prove this combination as a viable therapeutic option in the conservative management of this prevalent condition. The strengths of the study are the design and the novelty of the combination of methods. The main limitation is the short follow-up course.

Ethics and dissemination: The study is registered in the Clinical Trials database (protocol number: 8094833648737701) and was approved by the University Ethics Committee (number: 1373481).

Trial registration number: 8094833648737701 (NCT02757664); Pre-results.

\section{INTRODUCTION}

Calcaneus tendinopathy can be classified according to its anatomic site, as insertional and non-insertional tendinopathy. It is characterised by intratendinous degenerations, 
secondary to low grade inflammatory signs and erratic biological healing. ${ }^{1-3}$ Insertional tendinopathy occurs in the Achilles attachment to the tuberosity of the calcaneus bone and up to $2 \mathrm{~cm}$ proximal to the tuberosity. It is generally associated with a traction enthesophyte (upper spur), Haglund deformity ( pump bump) and with preAchilles bursitis and retroAchilles bursitis. The diagnosis is made based on clinical evaluation; ancillary tests, such as X-ray and ultrasound, are performed only to confirm the lesion and to exclude differential diagnoses (stress fractures, tumours). The clinical diagnosis consists of checking the level of pain via palpation of the tendinous insertion region in the calcaneus bone (and up to $2 \mathrm{~cm}$ around this region). The occurrence of volume increase and mild hyperaemia also supports the diagnosis. $^{1-4}$

Historically, the condition's initial treatment is based on eccentric strengthening of the tendon. Results for non-insertional tendinopathy are encouraging, with an $82 \%$ success rate when analysing return to previous activities. $^{5} 6$ However, evidence indicates that eccentric strengthening for insertional tendinopathy produced a rate of improvement ranging between $32 \%$ and $67 \%$ of the patients. ${ }^{2-4}$ Within this context, shock wave therapy has been proposed as a viable option, in case of failure of the conservative treatment and prior to referral to surgery. ${ }^{7-9}$ Over the past 30 years, extracorporeal shock waves have been safely and efficiently used in the treatment of various pathological conditions. ${ }^{10}{ }^{11}$ Extracorporeal shock wave lithotripsy (ESWL), for example, is a well-established treatment for urological pathologies. More recently, low ${ }^{12-14}$ and high ${ }^{14}{ }^{15}$ energy shock wave therapies are being used in the treatment of pseudoarthrosis ${ }^{16}{ }^{17}$ and several types of tendinopathy with prominent results. ${ }^{18-21}$

Accumulating evidence indicates that the use of shock waves induces neovascularisation and release of angiogenic markers by the recruitment of mesenchymal stem cells. ${ }^{12} 1322$ The molecular mechanism explaining how the shock wave produces these consequences is yet to be determined. ${ }^{15}{ }^{17}$ Neovascularisation improves blood irrigation, which, in its turn, contributes to tissue regeneration in the tendon-bone junction. Separate lines of inquiry suggested that shock wave therapy, relative to placebo therapies, induces a higher increase of mechanical resistance and concentration of markers of collagen synthesis (ie, hydroxiproline and pyridinoline), which are important components of the healing process. ${ }^{12} 1416$

Clinically, few complications are associated with shock waves, with transient regional hyperemia being the most frequently reported. Few patients $(5 \%)$ report having pain after high energy shock wave application, which normally ceases at the end of the treatment. ${ }^{8}$ Tendon rupture was described in the literature in only $1 \mathrm{study},{ }^{23}$ which showed 2 cases of older patients in a population of 49 cases. The authors could not find a true relation between the therapy and the events. Rasmussem et $a l^{24}$ have carried out a randomised clinical trial with 48 patients and 12 months of follow-up, comparing the use of radial shock wave therapy in patients after 4 weeks of conservative treatment, including stretching and strengthening, with the placebo. Superior results regarding pain and function were shown in the group that received the intervention. Kearney et al ${ }^{4}$ did a systematic review of the literature, looking for evidences concerning the calcaneus insertional tendinopathy treatment. They found only one paper ${ }^{8}$ with the usage of the high energy therapy, but, nevertheless, the work was criticised by the small sample and the methodology inconsistencies.

Shock wave therapy has been progressively more studied. Recent evidence has indicated that this technique is a promising option for the management of chronic insertional tendinopathy; however, the evidence is still insufficient to inform a consensus regarding the indication of this treatment in this very frequent condition. $^{9} 1925$ Our objective is to evaluate the effectiveness of low energy shock wave therapy associated with an eccentric strengthening protocol, and compare it to eccentric strengthening associated with placebo, using the function by the Victorian Institute of Sports Assessment-Achilles (VISA-A). The primary hypothesis is that adjunctive shock wave therapy will mitigate pain and improve function as compared to placebo.

\section{MATERIAL AND METHOD}

\section{Design, setting and recruitment}

This will be a double blind, placebo-controlled, parallel groups, randomised clinical trial (figure 1). The study will be conducted at Hospital São Paulo, a tertiary teaching hospital fully affiliated with the Universidade Federal de São Paulo (UNIFESP), in the Orthopedics and Traumatology Department (DOT) at the Centre of Tissue Research and Regeneration (CPRT).

Participants will be enrolled at the CPRT, which provides assessment and treatment to $\sim 10$ new patients with chronic insertional tendinopathy per week. They will be referred by local orthopaedist doctors or health professionals. Information to these physicians will be delivered by email addressed directly to them, as well as via posters exhibited in places containing orthopaedic medical care (outpatient clinic, emergency room).

\section{Inclusion criteria}

- Individuals must be older than 18 and younger than 65 years of age, both genders;

- Participants must be experiencing pain symptoms in the calcaneus tendon insertion region over the past 3 months;

- Clinical diagnosis of chronic insertional tendinopathy, defined as presence of pain at palpation of the tendinous insertion region in the calcaneus bone (and up to $2 \mathrm{~cm}$ around this region); and the occurrence of increased regional volume, associated with findings of tendinopathy in the ultrasound scan. 


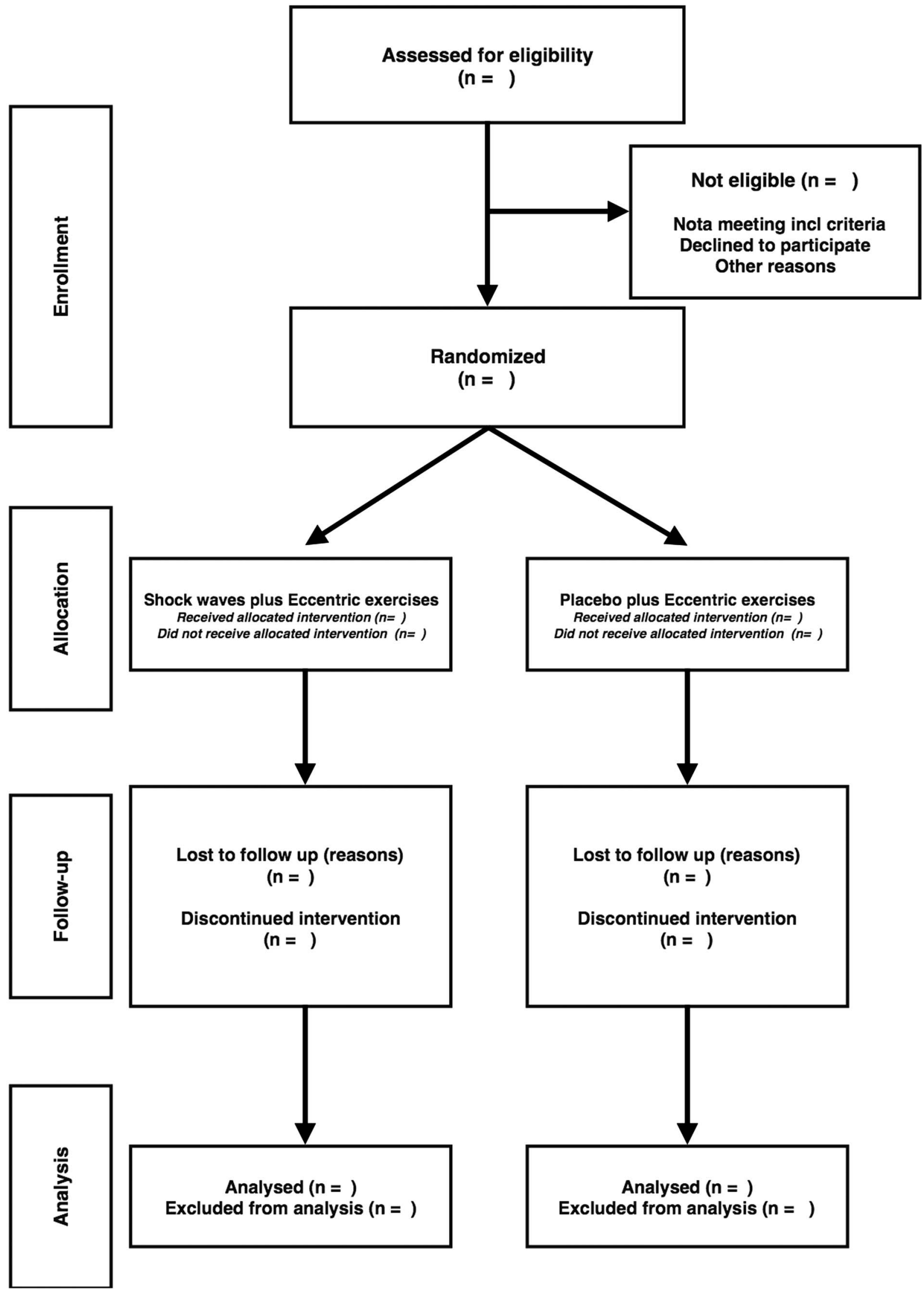

Figure 1 Flow_Achilles Project submitted to government funding (CNPq (Conselho Nacional de Pesquisa-National Research Council under the protocol number 8094833648737701$)$ ). 


\section{Exclusion criteria}

- Previous surgery involving the affected foot or ankle;

- History or documented evidence of autoimmune or peripheral vascular diseases;

- History or documented evidence of peripheral neuropathy (nervous compression syndrome, tarsal tunnel syndrome) or systemic inflammatory disease (rheumatoid arthritis, spondylitis, Reiter syndrome, etc);

- Non-insertional or mixed tendinopathy (insertional and non-insertional);

- Previous infiltration in the affected tendon over the 6 months preceding the initial assessment;

- Beginning of the present pain, due to a trauma;

- Pregnancy;

- Any condition that represents a contraindication of the proposed therapies;

- Impossibility or incapacity to sign the informed consent form;

- History or documented evidence of blood coagulation disorders (including treatment with anticoagulants, but excluding aspirin);

- Use of heart pacemaker;

- Presence of infectious process (superficial on skin and cellular tissue, or deep in the bone) in the region to be treated;

\section{Sampling}

The sampling calculation is based on the studies of Rompe et $a l^{21}$ and Sayana $e t a l,{ }^{6}$ and considers an estimated effect size of 3.3, a SD of 16.2 and sampling error of $5 \%$. It was calculated considering 93 patients divided into 2 groups in a randomised way, estimating that 41 evaluable participants per treatment arm will have better than $80 \%$ power to detect a difference in results between the shock wave and the placebo participants at a level of $5 \%$ significance. Since we expected a $10 \%$ loss in the follow-up, based on other clinical studies, we plan to include an extra $10 \%$ of participants, totalling 51 patients per group.

\section{Procedures}

A written, signed and dated informed consent will be obtained from the participant before any study-related procedures are performed. The patients will have to fill out an initial questionnaire in order to be enrolled (Attachment 1). After that, the assistant doctor will do the physical diagnostic examination. Then ultrasound and X-ray procedures will take place, in order to complete the diagnostic assessment. The patient will be included in the protocol and duly randomised after the diagnostic confirmation via anamnesis and physical examination, and also after completion of supplementary tests, as well as fulfilment of all the inclusion criteria and non-adequacy to the exclusion criteria.

The randomisation sequence will be generated via computing software (http://www.randomizer.org/form. $\mathrm{htm})$, producing a list from $1-\mathrm{x}$, and each number will be related to a sole treatment method. We will do a randomisation with interchanged blocks, with the same number of patients in each group.

Each non-transparent, opaque, sealed envelope, numbered from 1 to 104, will contain either a paper with the word 'physiotherapy' or with the words 'physiotherapy and shock wave'. Each treatment method will have the same number of envelopes. The patients will be initially assessed individually, being randomised and allocated in the same way. The intervention procedures will be the same, with the same positioning and preparations, but differing regarding the existence of a support at the applicator head of the shock wave apparatus in the group of patients without shock wave.

Neither the patient nor the evaluator doctor will have access to the protocol test applied to each patient, and the shock wave (or the placebo) will be conducted by a different physician. The patients in the placebo group will receive an apparatus' therapeutic head with the support that impedes the shock wave propagation directly on the appliance field. This will prevent the insertional region from receive any healing stimulus. Patients will still be able to hear the equipment's shock wave noise and feel the tremble provoked by the machine in contact with the heel.

\section{Intervention}

\section{Usage of shock waves}

1. Period from diagnosis to intervention: up to 1 week.

2. The patient will be lying on the stretcher in the supine position; barefooted, with ear muffs, the feet towards the shock wave apparatus.

3. The procedure region will be marked with ink (tendon insertion: the point with the highest local bulging or the penultimate transversal crease of the skin in the region).

4. Ultrasound gel will be applied on the region that will receive the shock wave;

5. Radial shock waves will be applied with a BLT600 equipment (BTL Medical Technologies-Canada), the intensity being $2000-3000$ pulses, $7-10 \mathrm{~Hz}$ of frequency, and 1,5 a 2,5Bar of intensity per application.

6. Shock waves will be applied on the first day of treatment (D0) as described above, then repeated on the second week after the first intervention (2nd week) and 4 weeks after the first intervention (4th week).

\section{Group without shock wave}

1. Period from diagnosis to intervention: up to 1 week.

2. Patient lying on the stretcher in the supine position; barefooted, with ear muffs, the feet towards the shock wave apparatus.

3. Localisation of the procedure region, marking it with ink (tendon insertion: the point with the highest local bulging or the second last transversal crease of the skin in the region).

4. Appliance of US gel on the region that will receive the shock wave; 
5. Placing of the apparatus' therapeutic head with the support that impedes the shock wave propagation directly on the appliance field.

6. Appliance of radial shock waves with the BLT600 equipment (BTL Medical Technologies-Canada), the intensity being $2000-3000$ pulses, $7-10 \mathrm{~Hz}$ of frequency, and 1,5 a $2,5 \mathrm{Bar}$ of intensity per application.

7. Appliance on the first day of treatment (D0) as described above, repeated on the second week after the first intervention (2nd week) and 4 weeks after the first intervention (4th week).

\section{Eccentric exercises}

The groups will be submitted to the Alfredson eccentric strengthening protocol ${ }^{26-28}$ for 12 weeks, starting on the same day of the first appliance. The exercises will be shown to the patients by the assistant doctor, and a booklet (Attachment 2) will be handed out, with detailed explanation concerning the protocol to be followed. The patient will practise the exercises standing on ground level, starting from a flexed ankle position (tiptoes). Participants will do exercises of passive ankle extension (dorsiflexion), 3 series of 15 repetitions, with the knee stretched and 3 series of 15 repetitions with the knee flexed at $20^{\circ}$. The eccentric stage (downwards) of the movement will be carried out slowly, while the patient contracts the muscles and increases the distance between the attachment and insertion points. This must be performed only with the affected member, until its heel reaches the terrain level. The concentric stage (upwards) will be carried out only with the non-affected member. In case the pathology involves two members, the patient will use the upper members to help the practice in the concentric stage. The patients will be encouraged to increase the load with $5 \mathrm{~kg}$ load weights placed in a backpack which the patient will wear to practise the exercise. The load is increased gradually as long as the exercise is painless to the patient. The objective's fulfilment and the quality of the exercise are indicated by the discomfort felt on that region after the performance of the series.

The patients cannot perform their base sports activities during the first 8 weeks of training. After the fourth week, they will be free to run on a flat incline, as well as to do biking and water activities that do not generate painful symptoms. After the eighth week, they will be permitted to gradually restart the sports activities that they used to perform previously, as long as they are not feeling any pain. The strengthening exercise, the intensification process, as well as the engagement in the treatment will be checked during the return to repeat the appliances; and during the follow-up with the doctor. To increase adhesion to the intervention protocol, handouts with tables containing dates indicating the days the patient executes the protocol's exercises; also with blank spaces for notes about the use of medication or occurred complications.

\section{Adjuvant therapies}

Both groups will be submitted to the same post intervention care programme, and they will be advised to use the following adjuvant therapies according to the intensity of their symptoms:

\section{Cryotherapy}

Every patient will be oriented to perform cold compresses on the tendon insertional region three times a day, during $20 \mathrm{~min}$, with at least 2 hours of interval between them.

\section{Painkillers}

Level 1:

- Dipyrone $1 \mathrm{~g}$ every 6 hours, in case of pain, or

- Paracetamol $750 \mathrm{mg}$ every 6 hours

Level 2 (in case the pain does not diminish with level 1):

- Tramadol $50 \mathrm{mg}$ every 6 hours, in case of pain, or

- Codeine $30 \mathrm{mg}$ every 6 hours, in case of pain.

The patient must present, at each visit to the doctor, the daily annotation concerning the used sedative medication. In case the pain increases right after any of the established treatments, the patient will be permitted to take analgesics (group 1) during a period of 5 days. The medication will be supplied to the patient after the intervention, with the respective orientation concerning its use. After the period of 5 days of sedation, in case the pain persists, the patient will be reassessed, to check the necessity of changing the medication (group 2). If after the second assessment (with 6 weeks) the pain is stronger than in the initial painful stage (prior to the treatment), the patient will have the option of either changing the treatment or being excluded from the study.

\section{Primary outcome}

- Visa-A Score

Significant increase in the studied group's score in comparison to the preintervention scores.

\section{Secondary outcome}

- Visual Analogue Scale

- American Orthopedic Foot and Ankle Society scale

- Foot and Ankle Outcome Score

- Short Form Health Survey 12-item

- Algometry (pain threshold and visual analogue scale with $3 \mathrm{~kg}$ )

\section{Participant discontinuation}

Participants may be discontinued from the study participation at any time. Reasons for discontinuation include:

1. Voluntary discontinuation by the participant without prejudice to further treatment.

2. Development of complex regional pain syndrome or any huge inflammatory response. Achilles tendon rupture (all of them are going to be considered failure).

3. Pain and function severe impairment. 


\section{Strategies to increase adhesion to intervention protocols}

Handouts with tables containing dates indicating the days the patient executes the protocol's exercises and also having blank spaces for notes about the use of medication or occurred complications.

\section{Statistical analysis}

Primary analysis will be performed on the intentionto-treat population (all participants with at least one study intervention and one postbaseline efficacy assessment). The primary point for analyses of efficacy will be week 24. The Mixed-Model for Repeated Measures method will be used to impute missing data for participants who discontinue during the study. The primary efficacy measure will be change from baseline to study end point on the Visa-A Score, which will be analysed with a repeated measures t-test. Subsequently, repeated measures MANCOVA will be administered to test for covariations and main effects. The significance level will be set at a $p$ value $<0.05$.

\section{DISCUSSION}

Insertional Achilles tendinopathy is a common condition, affecting both athletes and the sedentary population. Its aetiology is related to a poor biological body response after micro lesions to the tendon (occurring during training or in a daily usual activity). Degenerative changes and a low inflammatory reaction are the characteristics of these tissues, revealing a low healing response to injury. This illness normally induces patients to look for medical care due to pain, function impairment and decrease in athletic performance. Approximately $16 \%$ of the active individuals end up abandoning their sports activities in consequence of this disorder.

The traditional initial treatment of choice is nonsurgical, comprehending modalities such as physical therapy and exercises. Yet this approach has not produced encouraging results over the past few years, and currently there is still no standard conservative treatment for Achilles insertional tendinopathy. Whereas the eccentric strengthening programme is one of the clinicians' preferred modalities of treatment, it has not led to the same good results as in other tendon locations. This scenario contributed to the increase in the number of surgeries performed for this illness in the past few decades. Procedures commonly associated to high costs and possible complications, such as wound dehiscence, infection, nerve damage and tendon rupture.

Several alternatives to the classical treatment (eg, infiltration, electrostimulation, sclerotherapy, among others) have been considered, in order to stimulate healing stimulus to the degenerated tendon. The low success rates have provided the impetus to explore practical and cheaper ways to induce adequate reparation conditions. By stimulating soft tissue healing by angiogenesis enhancing and diffusion of cytokine molecules, the shock wave therapy has become a reliable option in the treatment of this illness.

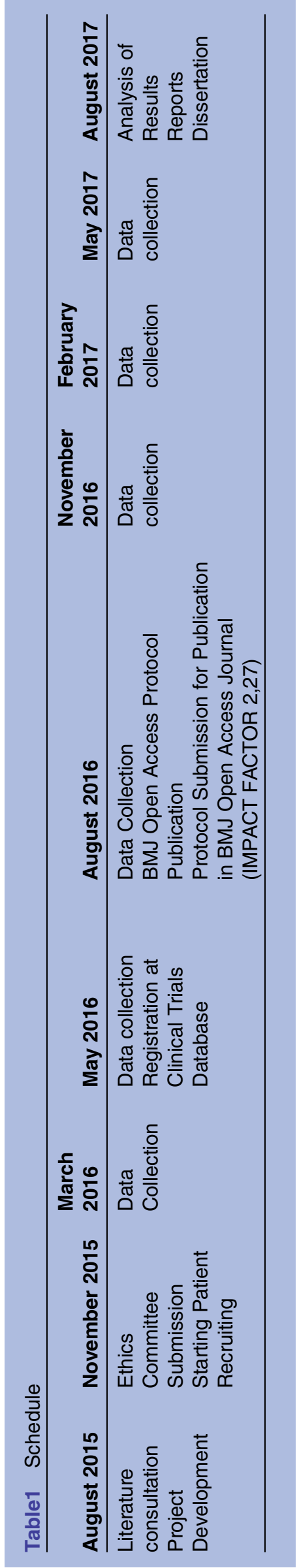


While isolated shock wave treatment has shown encouraging results during the past years, they were not definitive. Adjuvant administration of the Alfredson protocol proved to be a trustworthy combination in the noninsertional presentation of this disorder in a recent study. The technical composition of shock wave and tendon's eccentric strengthening can be the answer to the patient's improvement in the Achilles insertional tendinopathy, with the additional benefit of avoiding the complications and high costs associated with the surgical treatment.

\section{ETHICS AND MEANS OF DISSEMINATION}

The Project is registered in the Clinical Trials database under the protocol number 8094833648737701 (NCT02757664) on 05/02/2016. The study was approved by the University Ethics Committee under the number 1373481 (table 1).

\section{Author affiliations}

${ }^{1}$ Department of Orthopaedics, Universidade Federal de Sao Paulo, Sao Paulo, SP, Brazil

${ }^{2}$ Orthopedics and Traumatology—Division of Hand Surgery and Upper Limb, Federal University of São Paulo (UNIFESP/EPM), São Paulo, SP, Brazil

Contributors NSBM was the main researcher. FF and JCB participated in co-orientation and study design. SJMI participated in co-orientation, writing and study design. FTM took part in co-orientation, writing and data collecting. PRDidS and BSdS took part in shock-wave application and implementation. OLC participated in literature revision and writing. GP participated in Visa-A translation to Portuguese and implementation. BTA was the medical student enrolled in the project, took part in implementation and data collecting. MJST took part in main orientation, literature revision, writing, study design and paper submissions. All authors contributed to refinement of the study protocol and approved the final manuscript.

Funding Study being conducted in São Paulo Federal University (UNIFESP), São Paulo—SP, Brazil.

Competing interests None declared.

Ethics approval Sao Paulo Federal University Ethics Committee (protocol number: 1373481).

Provenance and peer review Not commissioned; externally peer reviewed.

Data sharing statement Results, technical appendix, statistical code and data set will be openly available through a DOI. Non-digital data supporting this study will be stored by the corresponding author at the Sao Paulo Federal University. Details of how to request access to these data will be provided in the final document.

Open Access This is an Open Access article distributed in accordance with the Creative Commons Attribution Non Commercial (CC BY-NC 4.0) license, which permits others to distribute, remix, adapt, build upon this work noncommercially, and license their derivative works on different terms, provided the original work is properly cited and the use is non-commercial. See: http:// creativecommons.org/licenses/by-nc/4.0/

\section{REFERENCES}

1. Den Hartog BD. Insertional Achilles tendinosis: pathogenesis and treatment. Foot Ankle Clin 2009;14:639-50.

2. Irwin TA. Current concepts review: insertional achilles tendinopathy. Foot Ankle Int 2010;31:933-9.

3. Magnan B, Bondi M, Pierantoni S, et al. The pathogenesis of Achilles tendinopathy: a systematic review. Foot Ankle Surg 2014;20:154-9.

4. Kearney R, Costa ML. Insertional achilles tendinopathy management: a systematic review. Foot Ankle Int 2010;31:689-94.
5. Sussmilch-Leitch SP, Collins NJ, Bialocerkowski AE, et al. Physical therapies for Achilles tendinopathy: systematic review and meta-analysis. J Foot Ankle Res 2012;5:15.

6. Sayana MK, Maffulli N. Eccentric calf muscle training in non-athletic patients with Achilles tendinopathy. J Sci Med Sport 2007;10:52-8.

7. Al-Abbad H, Simon JV. The effectiveness of extracorporeal shock wave therapy on chronic achilles tendinopathy: a systematic review. Foot Ankle Int 2013;34:33-41.

8. Furia JP. High-energy extracorporeal shock wave therapy as a treatment for insertional Achilles tendinopathy. Am J Sports Med 2006;34:733-40.

9. Furia JP. [Extracorporeal shockwave therapy in the treatment of chronic insertional Achilles tendinopathy]. Orthopade 2005;34:571-8. German.

10. Maffulli G, Hemmings S, Maffulli N. Assessment of the Effectiveness of Extracorporeal Shock Wave Therapy (ESWT) For Soft Tissue Injuries (ASSERT): an Online Database Protocol. Transl Med UniSa 2014:10:46-51. eCollection 2014 Sep.

11. Giusti Guilherme, Penteado Fernando Travaglini, Baptista Gomes dos Santos João, et al. Efeito de ondas de choque na placa de crescimento de coelhos. Acta ortop bras 2005[cited 2016 Oct 17];13:31-4. Available from: http://www.scielo.br/scielo.php? script=sci_arttext\&pid=S141378522005000100008\&lng=en .

12. Wang CJ, Huang $\mathrm{HY}$, Pai $\mathrm{CH}$. Shock wave-enhanced neovascularization at the tendon-bone junction: an experiment in dogs. J Foot Ankle Surg 2002;41:16-22.

13. Wang CJ, Wang FS, Yang KD, et al. Shock wave therapy induces neovascularization at the tendon-bone junction. A study in rabbits. J Orthop Res 2003;21:984-9.

14. Hsu RW, Hsu WH, Tai CL, et al. Effect of shock-wave therapy on patellar tendinopathy in a rabbit model. $J$ Orthop Res 2004;22:221-7.

15. Chen YJ, Wang CJ, Yang KD, et al. Extracorporeal shock waves promote healing of collagenase-induced Achilles tendinitis and iEEEEEncrease TGF-beta1 and IGF-I expression. J Orthop Res 2004;22:854-61.

16. Chen YJ, Kuo YR, Yang KD, et al. Shock wave application enhances pertussis toxin protein-sensitive bone formation of segmental femoral defect in rats. $J$ Bone Miner Res 2003;18:2169-79.

17. Wang CJ, Chen HS, Chen CE, et al. Treatment of nonunions of long bone fractures with shock waves. Clin Orthop Relat Res 2001 (387):95-101.

18. Mani-Babu S, Morrissey D, Waugh C, et al. The effectiveness of extracorporeal shock wave therapy in lower limb tendinopathy: a systematic review. Am J Sports Med 2015;43:752-61.

19. Hart L. Shock-wave treatment was more effective than eccentric training for chronic insertional achilles tendinopathy. Clin J Sport Med 2009;19:152-3.

20. Rompe JD, Nafe B, Furia JP, et al. Eccentric loading, shock-wave treatment, or a wait-and-see policy for tendinopathy of the main body of tendo Achillis: a randomized controlled trial. Am J Sports Med 2007:35:374-83.

21. Rompe JD, Furia J, Maffulli N. Eccentric loading versus eccentric loading plus shock-wave treatment for midportion achilles tendinopathy: a randomized controlled trial. Am J Sports Med 2009;37:463-70.

22. Notarnicola A, Moretti B. The biological effects of extracorporeal shock wave therapy (eswt) on tendon tissue. Muscles Ligaments Tendons J 2012;2:33-7. Print 2012 Jan.

23. Costa ML, Shepstone L, Donell ST, et al. Shock wave therapy for chronic Achilles tendon pain: a randomized placebo-controlled trial. Clin Orthop Relat Res 2005;440:199-204.

24. Rasmussen S, Christensen M, Mathiesen I, et al. Shockwave therapy for chronic Achilles tendinopathy: a double-blind, randomized clinical trial of efficacy. Acta Orthop 2008;79:249-56

25. Rompe JD, Furia J, Maffulli N. Eccentric loading compared with shock wave treatment for chronic insertional achilles tendinopathy. A randomized, controlled trial. J Bone Joint Surg Am 2008;90:52-61.

26. Mafi N, Lorentzon R, Alfredson $\mathrm{H}$. Superior short-term results with eccentric calf muscle training compared to concentric training in a randomized prospective multicenter study on patients with chronic Achilles tendinosis. Knee Surg Sports Traumatol Arthrosc 2001;9:42-7.

27. Fahlström $\mathrm{M}$, Jonsson $\mathrm{P}$, Lorentzon $\mathrm{R}$, et al. Chronic Achilles tendon pain treated with eccentric calf-muscle training. Knee Surg Sports Traumatol Arthrosc 2003;11:327-33.

28. Ohberg L, Alfredson $\mathrm{H}$. Effects on neovascularisation behind the good results with eccentric training in chronic mid-portion Achilles tendinosis? Knee Surg Sports Traumatol Arthrosc 2004;12:465-70. 